\title{
Dysregulation of the Microvasculature in Nonlesional Non-Sun- exposed Skin of Patients with Lupus Nephritis
}

\author{
PETER M. IZMIRLY, MD, MARIANNA SHVARTSBEYN, MD, SHANE MEEHAN, MD, ANDREW \\ FRANKS, MD, FACP, ALAN BRAUN, MD, ELLEN GINZLER, MD, SHERRY X. XU, PhD, \\ HERMAN YEE, MD, TANIA L. RIVERA, MD, CHARLES ESMON, PhD, LAURA BARISONI, \\ MD, JOAN T. MERRILL, MD, JILL P. BUYON, MD, and ROBERT M. CLANCY, PhD \\ From the Department of Medicine, Department of Pathology, and Department of \\ Dermatopathology, New York University (NYU) School of Medicine, New York, New York; Mercy \\ Hospital, Des Moines, lowa; Downstate Medical Center, State University of New York (SUNY), \\ New York, New York; and Oklahoma Medical Research Foundation, Oklahoma City, Oklahoma, \\ USA
}

\begin{abstract}
Objective-Membrane endothelial protein C receptor (mEPCR) is highly expressed in peritubular capillaries of kidneys from patients with active and poorly responsive lupus nephritis (LN). We investigated the hypothesis that changes in the microvasculature are widespread with extension to the dermal vasculature.
\end{abstract}

\begin{abstract}
Methods-Skin biopsies from uninvolved skin (buttocks) were performed in 27 patients with LN and 5 healthy controls. Sections were stained with specific antibodies reactive with mEPCR, adiponectin, intercellular adhesion molecule-1 (ICAM-1), and CD31; then assessed by enumeration of stained blood vessels (percentage positive blood vessels) blinded to knowledge of clinical information.
\end{abstract}

\begin{abstract}
Results-There was a significant increase in the prevalence of blood vessels that stained for mEPCR and ICAM-1 in patients compared to controls [94\% vs 59\% $(\mathrm{p}=0.045)$ and $81 \%$ vs $67 \%$ $(\mathrm{p}=0.037)$, respectively]. Adiponectin staining and CD31 staining were similar between the groups (45\% vs $43 \%$ and $98 \%$ vs $92 \%$ ). Dermal staining for mEPCR was greater in patients with proliferative glomerulonephritis than in those with membranous disease (96\% vs 60\%; $p=0.029$ ). A composite of poor prognostic renal markers and death was significantly associated with greater expression of mEPCR staining.
\end{abstract}

The Journal of Rheumatology Copyright @ 2012 . All rights reserved.

Address correspondence to: R. Clancy, NYU School of Medicine, Tisch Hospital Tch 4-407, 560 First Avenue, New York, NY 10016, USA. Bobdclancy@aol.com.

P.M. Izmirly, MD, Assistant Professor of Medicine, Department of Medicine, NYU School of Medicine; M. Shvartsbeyn, MD, Department of Pathology, NYU School of Medicine; S. Meehan, MD, Assistant Professor, Director, Dermatopathology, Department of Dermatopathology, NYU School of Medicine; A. Franks, MD, FACP, Clinical Professor of Dermatology and Medicine (Rheumatology), Director, Skin, Lupus and Autoimmune Connective Tissue Disease Section, Department of Medicine, NYU School of Medicine; A. Braun, MD, Mercy Hospital; E. Ginzler, MD, Distinguished Teaching Professor of Medicine, Downstate Medical Center, SUNY; S.X. Xu, PhD; H. Yee, MD, Associate Professor of Pathology; T. Rivera, MD, Department of Medicine, NYU School of Medicine; C. Esmon, PhD, Oklahoma Medical Research Foundation; L. Barisoni, MD, Department of Medicine, NYU School of Medicine; J.T. Merrill, MD, Program Chair, Clinical Pharmacology Research Program, Oklahoma Medical Research Foundation, Professor, Department of Medicine, University of Oklahoma Health Sciences Center; J.P. Buyon, MD, Professor of Medicine; R.M. Clancy, PhD, Associate Professor of Medicine, Department of Medicine, NYU School of Medicine. 
Conclusion-These data are consistent with the notion that in patients with $\mathrm{LN}$, activation of the microvasculature extends beyond the clinically targeted organ. The insidious expression of this widespread vasculopathy may be a contributor to longterm comorbidities.

\section{Key Indexing Terms}

\section{SYSTEMIC LUPUS ERYTHEMATOSUS; SKIN BIOPSY; LUPUS NEPHRITIS VASCULOPATHY; GLOMERULAR FILTRATION RATE; PROTEINURIA}

The endothelium is considered both the conduit and the boundary between blood-borne defense and potential inflammatory mediators and the cellular components of tissues. In addition to its role as a biologic sentinel, the endothelium dictates the effectiveness of blood flow critical to well-being of the tissues. Vascular perturbation in patients with systemic lupus erythematosus (SLE) is supported by the finding of elevated levels of soluble endothelial protein $\mathrm{C}$ receptor (EPCR), a soluble form of an integral membrane protein that regulates the conversion of protein $\mathrm{C}$ to activated protein $\mathrm{C}$, in patients compared to healthy controls, particularly those with active lupus nephritis $(\mathrm{LN})^{1,2}$. Further studies revealed that expression of membrane EPCR (mEPCR) was significantly increased in the cortical peritubular capillaries of kidneys from patients with biopsyproven nephritis compared to normal kidneys ${ }^{3}$. Upregulation of mEPCR was observed even in areas without tubulointerstitial damage. Of clinical relevance, elevated mEPCR staining was associated with a poor response to therapy. While this observation was initially unexpected given the prediction that shed EPCR would result in diminished mEPCR, it was reasoned that the increased expression represented endothelial activation in an attempt at repair. Thus, evidence of a potential vascular contribution to renal injury/damage was established.

The extent of vascular activation beyond the renal microvasculature to more readily accessible yet clinically silent organs, such as the dermis, may have relevance to the overall vascular well-being of patients with SLE. Two decades ago, Belmont and colleagues reported a significant lupus flare-dependent upregulation of proinflammatory molecules in the dermal blood vessels of nonlesional, non-sun-exposed (buttock) skin from patients with active SLE ${ }^{4,5}$. Specifically, levels of adhesion and proinflammatory molecules [vascular cell adhesion molecule-1, intercellular adhesion molecule (ICAM-1), E selectin, and nitric oxide synthase-2] assessed by immunohistochemistry (IHC) were found to be significantly elevated in patients compared to healthy controls. While these findings supported diffuse activation of the microvasculature in patients with SLE, attempts to use skin biopsies as an adjunct "factor" reflecting tissue reactivity in evaluating LN per se have not been reported. Moreover, there has been no assessment of other proteins with potential protective roles. One such candidate is adiponectin, an adipokine often associated with downregulation of adhesion molecule expression ${ }^{6,7}$. Adiponectin has been found in blood vessel walls after experimental endothelial injury ${ }^{8}$, and it is strongly expressed around infarcted but not normal myocardium ${ }^{9}$, supporting a role for it in vascular and endothelial recovery after insult.

Our study addressed the hypothesis that changes in the microvasculature extend beyond the clinically targeted organ and that dysregulation is a pathologic signature of SLE. The kidney 
was selected as the diseased organ since it represents one of the major organ system manifestations, associated with considerable morbidity and mortality ${ }^{10}$. Biopsies of nonlesional skin were chosen as a proxy in vivo source to gauge the status of the extrarenal microvasculature. Such evaluation might reveal a novel factor reflecting tissue reactivity for evaluation and treatment of patients with $\mathrm{LN}$, and also a potential pathologic link between the association of renal disease and vasculopathy. Patients with LN were recruited for skin biopsies of their buttocks; the majority of these were close to the time of renal biopsies.

\section{MATERIALS AND METHODS}

\section{Subjects}

The study protocol and informed consent were approved by the Institutional Review Board of New York University (NYU) School of Medicine. Twenty-seven patients met the following inclusion criteria: (1) confirmation of a diagnosis of SLE by the criteria of the American College of Rheumatology ${ }^{11}$; (2) confirmation of a diagnosis of $\mathrm{LN}$ based on renal biopsy ( $n=26$ ) or solely on proteinuria and active sediment $(n=1)$; (3) consent to undergo skin biopsy of the buttock; and (4) access to medical records. Skin was obtained from the buttocks ( $2 \mathrm{~mm}$ punch biopsy).

Two physicians (JPB, PMI) evaluated the patients and reviewed all medical records, while blinded to the skin biopsy staining results. Twenty-two patients were considered to have active nephritis at the time of skin biopsy as defined by protein/creatinine ratio $>500 \mathrm{mg} / \mathrm{g}$ creatinine with a subdivision of nephrotic and subnephrotic proteinuria, with values $>3 \mathrm{~g}$ and $<3 \mathrm{~g}$, respectively. Skin biopsies were obtained within 1 month of the renal biopsy in 14 patients, within 6 months in 4 patients, within 9 months in 1 patient, and within 3 years in 2 patients; and a renal biopsy was refused by 1 patient. Five patients had inactive nephritis [normal glomerular filtration rate (GFR), proteinuria $<500 \mathrm{mg} / \mathrm{g}$ creatinine, and no urinary red blood cell casts, but with history of renal disease based on biopsies done within 2 years of the skin biopsy]. Each subject was scored using a weighted composite (range 0-15) of the following: (1) death within 2 years of the skin biopsy (0 or 5); (2) GFR $<60 \mathrm{ml} / \mathrm{min}$ (0 or 4); (3) proteinuria $>3 \mathrm{~g} / 24 \mathrm{~h}(0$ or 3$)$; (4) duration of nephritis $>1$ year at time of skin biopsy ( 0 or 2); and (5) abnormally low $\mathrm{C} 3$ or $\mathrm{C} 4$ (0 or 1$)$. Clinical variables were selected from a panel of biomarkers that were independently predictive of renal response to therapy at 6 months for patients from the Aspreva Lupus Management study (ALMS) ${ }^{12}$. There were 5 healthy controls, who were faculty/staff employees of NYU School of Medicine.

\section{Immunohistochemical staining of skin sections}

The immunostaining was performed using a validated protocol with a "positive" blood vessel by IHC reflecting the intensity of positive stain $\geq 2+$ (range 0 to 3 ). Briefly, paraffinembedded skin sections were individually stained with specific antibodies against mEPCR (monoclonal antibody, HEPCR 1489 provided by C. Esmon; 1:100), rabbit anti-human adiponectin (Thermo Scientific, Pittsburgh, PA, USA; no. PA1-84881), rabbit anti-human ICAM-1 (Cell Signaling Technology, Beverly, MA, USA; no. 4915), and CD31 (positive control, pan-endothelial marker; no. 014-1096-050; Celerus Diagnostics, Carpinteria, CA, USA). Mouse IgG and rabbit IgG (Southern Biochemicals, Birmingham, AL, USA) were 
used as negative controls. Secondary antibodies included biotinylated anti-mouse IgG (Vector Laboratories, Burlingame, CA, USA; no. BA-2000) and biotinylated anti-rabbit IgG (Vector Laboratories, no. BA-1000). Slides were developed with ABC reagents and counterstained. IHC was scored based on the intensity of the staining and the percentage of staining blood vessels, calculated as a ratio of peroxidase brown-labeled vessels divided by the total number of vessels in the sections examined. IHC results were analyzed by 2 independent pathologists (SM, MS, with assistance of RC) without knowledge of the clinical information associated with the biopsy. The data are reported as a reader average mean of the percentage of positive-staining blood vessels (> 2+, scale $0-3)$, which were normalized to the total blood vessel count.

\section{Statistical analysis}

Median values of the percentage of positive-staining blood vessels are reported. MannWhitney test for unpaired data was used to compare percentage positive-staining blood vessels for each endothelial marker between patients and controls. Fisher's exact test was performed to evaluate associations between categorical variables. A 2-sided $\mathrm{p}$ value $<0.05$ was considered significant.

\section{RESULTS}

\section{SLE patients and controls}

Demographic data of the 27 patients with SLE and 5 healthy controls are shown in Table 1. Both groups were ethnically diverse, whites representing $26 \%$ of patients with SLE and $40 \%$ of controls. As expected, C3 and C4 levels were low in 73\% of patients and normal in all controls. Abnormal titers of anti-dsDNA antibodies were present in $77 \%$ of patients with SLE and none of the controls. With regard to International Society of Nephrology/Renal Pathology Society (ISN/RPS) biopsy class for LN, 15 had Class IV (58\%), 2 Class III (8\%), 7 Class V (27\%), and 1 mixed membranoproliferative Class III, V (4\%). One biopsy had diffuse immune complex deposition throughout the tubulointerstitial compartment associated with a dense inflammatory infiltrate and interstitial fibrosis. In addition, there were sclerotic glomeruli with fibrous crescents. This was considered acute, chronic interstitial LN (4\%). There was a trend to lower GFR in patients than in controls $(84 \mathrm{ml} / \mathrm{min}$ vs $98 \mathrm{ml} / \mathrm{min}$, respectively) and 4 patients (15\%) had GFR $<60 \mathrm{ml} / \mathrm{min}$. Ten patients (37\%) had proteinuria in the nephrotic range.

\section{Evaluation of endothelial candidate factors in the skin biopsies from patients with SLE and controls}

The median percentage of mEPCR-positive staining of the nonlesional non-sun-exposed dermal blood vessels was significantly higher in the patients with SLE compared to controls ( $94 \%$ vs 59\%; $p=0.046$; Table 1). The median percentage of ICAM-1-positive staining was also significantly higher in SLE patients compared to controls ( $81 \%$ vs $67 \% ; \mathrm{p}=0.037)$.

Representative immunostains of mEPCR and ICAM-1 from a patient with active LN and a healthy control are shown in Figure 1. With regard to mEPCR, 100\% of the blood vessels were positive from a patient with Class IV disease (Figure 1, upper left panel), who had abnormal complement and abnormal anti-dsDNA, and who died 18 months after the skin 
biopsy; only $13 \%$ of vessels were positive in the control (lower left panel). With regard to ICAM-1, 98\% of blood vessels were positive in the patient (upper right panel) and $22 \%$ in the control (lower right panel).

In contrast to mEPCR and ICAM-1, there were no differences in the median percentage of staining for adiponectin in the patients compared to controls ( $45 \%$ vs $43 \%$, respectively; $p=$ nonsignificant; Table 1). No differences were seen between the 2 groups in terms of staining for CD31, which served as a positive control (98\% vs 92\%; $\mathrm{p}=$ nonsignificant; Table 1).

\section{Association of mEPCR in dermal microvasculature with morbidity score, active nephritis, and biopsy class}

Endothelial reactivity in the dermal microvasculature was then assessed in relation to measures of mEPCR (percentage of positive blood vessels), renal activity, specific biopsy class, and renal prognostic composite (Table 2). As predicted by the overall comparison of patients with controls, specifically, the median percentage positive staining for mEPCR was significantly increased in patients with active nephritis ( $96 \%$ vs $59 \% ; p=0.028)$. There were significantly higher median levels of mEPCR percentage positive staining in patients with Classes III and IV disease and biopsies showing diffuse immune complex deposition in the tubulointerstitium (the immune complex deposition was in the subendothelium and microvascular bed) than in those with Class $\mathrm{V}$ disease (immune complex on the subepithelial side; $96 \%$ vs $60 \%$; $\mathrm{p}=0.029$ ).

Median values of mEPCR were significantly higher in patients with a renal prognostic composite score $>3$ (range $0-15)$ than in those with a score < $3(96 \%$ vs $83 \% ; p=0.036$; Table 2). In addition, an $\mathrm{mEPCR}>93 \%$ was associated with a poor prognostic renal score $>$ 3 in 11/14 patients compared to an mEPCR < 93\%, which was observed in 5/13 with a composite $<3(\mathrm{p}=0.021)$. Neither ICAM-1 nor adiponectin was associated with active nephritis, biopsy class, or renal prognostic score $>3$ (Table 2).

\section{DISCUSSION}

The microcirculation may hold valuable clues for better characterization and understanding of vascular injury in lupus patients in general and LN in particular. In our study, using immunohistological assessments of all dermal blood vessels in uninvolved non-sun-exposed skin, expression of mEPCR, ICAM-1, and adiponectin was detectable but the extent and intensity varied between the patients and healthy controls. There were significantly greater median levels of percentage of positively staining blood vessels for mEPCR and ICAM-1 in the patients compared to controls. This latter finding is consistent with a previous report ${ }^{4}$ that during nonrenal SLE flares, ICAM-1 is upregulated on endothelial cells in nonlesional buttock skin. Unique to our study is the identification of widespread upregulation of mEPCR in patients with renal disease, a finding that supports a role of mEPCR both in endothelial activation and in an attempt at repair. While the increased mEPCR expression previously reported in the kidney was associated with histologic features of tissue inflammation and injury $^{3}$, significantly increased endothelial expression of $\mathrm{MEPCR}$ in the dermal vessels was observed in histologically and clinically normal tissue. 
Immune complex deposition may in part account for the modulation of mEPCR and ICAM-1 reported here. However, in previous work by Belmont, et al adhesion molecules were upregulated in the non-sun-exposed nonlesional skin of patients with lupus, and neither immune globulins nor $\mathrm{C} 3$ were observed ${ }^{4}$. Moreover, in our study, the phenotype of blood vessels was determined in a wide zone of the dermis including the papillary dermis and reticular dermis. In contrast, the site of the traditional lupus band test is above the dermal papillary blood vessels.

While the stimulus accounting for increased mEPCR expression in patients with SLE is unknown, the coagulation protease thrombin is a strong candidate with regard to the microvasculature. While the endothelium is often regarded as 1 organ, the macrocirculation and microcirculation are best considered as separate entities. For example, in large vessels, thrombin promotes a prothrombotic state that is appropriate to its role in the prevention of catastrophic blood loss. In contrast, in the microcirculation, the pendulum swings and the ability of thrombin to clot blood is counterbalanced by several molecules including thrombomodulin, protein S, and mEPCR (for a recent review, see Esmon and Esmon ${ }^{13}$ ).

The effect of inflammation is broadly "sensed" by the microcirculation. An elastasemediated cleavage of throm-bomodulin ${ }^{14}$ may result in the loss of a key antithrombotic molecule, and active prothrombotic thrombin may increase locally and systemically. Thus, if elastase is released at an injury site, it will diffuse into the circulation and reach all of the microvasculature, including that of noninvolved skin. In addition, since thrombomodulin inhibits complement ${ }^{15}$, there may be a loss of a "brake" to complement activation, resulting in high levels of membrane ICAM-1 as described by Belmont, et al in the nonlesional skin ${ }^{4}$. In patients, while it is seemingly paradoxical, high levels of mEPCR expression in the dermal microvasculature of nonlesional skin and the peritubular capillaries of clinically and histologically diseased kidneys may each represent attempts of the microvasculature to restore baseline levels of antithrombotic thrombin. In support of this notion, the observed elevated mEPCR expression may highlight a pathologic condition that represents an important but previously unrecognized deficit of an "active protein C-dependent signaling sensitive" pathway ${ }^{16}$, which may explain the association with poor renal outcome ${ }^{3}$. Accordingly, distal injury may initiate effects on mEPCR expression in a patient's normal skin as a systemic response by the microcirculation to redistribute the endothelial cellderived "candidates" to achieve the common goals of an antithrombotic thrombin and homeostasis.

Data from Davidson and coworkers suggest that activated effector cells drive renal inflammation with early participation of the endothelium in leukocyte extravasation and infiltration ${ }^{17}$. Thus mEPCR may fill the "gap" to restore the microcirculation's antithrombotic capacity. In this hypothesis, $\mathrm{mEPCR}$ binds protein $\mathrm{C}$, presenting it to the thrombin/thrombomodulin complex, thus regulating its conversion to activated protein $\mathrm{C}^{18}$. In addition, activated protein $\mathrm{C}$ serves as a coreceptor of a complex with $\mathrm{mEPCR}$, and this complex binds to the protease-activated receptor-1 that promotes vascular integrity (i.e., it diminishes vascular leakage) and suppresses the release of thrombospondin- $1^{19}$, shown to induce apoptosis specifically in cytokine-activated angiogenic endothelial cells ${ }^{20}$. Considered together, the high expression of mEPCR may represent an attempt to preserve an 
antithrombotic state of the microvasculature or to generate "free" active protein $\mathrm{C}$ to nudge the endothelial phenotype toward one conferring protection. Regarding the finding that there were no differences between patients and controls in the expression of adiponectin, the simple interpretation is that while ICAM-1 and mEPCR are linked to a property of the microvasculature such as the regulation of thrombin, adiponectin does not have a similar role. No relationship with renal disease was evident.

One limitation of our study is that a few of the patients not unexpectedly had active cutaneous or musculoskeletal disease at the time of the skin and renal biopsy, thus tempering the unambiguous attribution of exclusively renal disease to the widespread endothelial dysfunction. Nevertheless, the findings confirm that SLE is a systemic disease, including at the endothelial level.

Patients with LN had increased mEPCR expression in the dermal microvasculature. This observation suggests that $\mathrm{mEPCR}$ expression in clinically inactive skin may represent a novel candidate factor reflecting tissue reactivity and functional dysregulation of the microcirculation in general. The insidious expression of this widespread vasculopathy may be a contributor to longterm comorbidities.

\section{Acknowledgments}

Supported by NIH/NIAMS grant R01 AR055088-01 to Dr. Clancy; and a Career Development Award to Dr. Izmirly by the SLE Lupus Foundation Inc., New York.

We thank Constantinos Cachimpanis for technical assistance in validation in the immunostaining protocols and Amanda Zink for help preparing this report.

\section{References}

1. Sesin CA, Yin X, Esmon CT, Buyon JP, Clancy RM. Shedding of endothelial protein C receptor contributes to vasculopathy and renal injury in lupus: In vivo and in vitro evidence. Kidney Int. 2005; 68:110-20. [PubMed: 15954900]

2. Clancy R, Ginzler EM. Endothelial function and its implications for cardiovascular and renal disease in systemic lupus erythematosus. Rheum Dis Clin North Am. 2010; 36:145-60. [PubMed: 20202596]

3. Izmirly PM, Barisoni L, Buyon JP, Kim MY, Rivera TL, Schwartzman JS, et al. Expression of endothelial protein $\mathrm{C}$ receptor in cortical peritubular capillaries associates with a poor clinical response in lupus nephritis. Rheumatology. 2009; 48:513-9. [PubMed: 19286697]

4. Belmont HM, Buyon J, Giorno R, Abramson S. Up-regulation of endothelial cell adhesion molecules characterizes disease activity in systemic lupus erythematosus. The Shwartzman phenomenon revisited. Arthritis Rheum. 1994; 37:376-83. [PubMed: 7510492]

5. Belmont HM, Levartovsky D, Goel A, Amin A, Giorno R, Rediske J, et al. Increased nitric oxide production accompanied by the up-regulation of inducible nitric oxide synthase in vascular endothelium from patients with systemic lupus erythematosus. Arthritis Rheum. 1997; 40:1810-6. [PubMed: 9336415]

6. Ouchi N, Kihara S, Arita Y, Okamoto Y, Maeda K, Kuriyama H, et al. Adiponectin, an adipocytederived plasma protein, inhibits endothelial NF-kappa-B signaling through a cAMP-dependent pathway. Circulation. 2000; 102:1296-301. [PubMed: 10982546]

7. Ouchi N, Kihara S, Arita Y, Maeda K, Kuriyama H, Okamoto Y, et al. Novel modulator for endothelial adhesion molecules: adipocyte-derived plasma protein adiponectin. Circulation. 1999; 100:2473-6. [PubMed: 10604883] 
8. Okamoto Y, Arita Y, Nishida M, Muraguchi M, Ouchi N, Takahashi M, et al. An adipocyte-derived plasma protein, adiponectin, adheres to injured vascular walls. Horm Metab Res. 2000; 32:47-50. [PubMed: 10741683]

9. Ishikawa Y, Akasaka Y, Ishii T, Yoda-Murakami M, Choi-Miura NH, Tomita M, et al. Changes in the distribution pattern of gelatin-binding protein of $28 \mathrm{kDa}$ (adiponectin) in myocardial remodelling after ischaemic injury. Histopathology. 2003; 42:43-52. [PubMed: 12493024]

10. Danila MI, Pons-Estel GJ, Zhang J, Vila LM, Reveille JD, Alarcon GS. Renal damage is the most important predictor of mortality within the damage index: Data from LUMINA LXIV, a multiethnic US cohort. Rheumatology. 2009; 48:542-5. [PubMed: 19233884]

11. Tan EM, Cohen AS, Fries JF, Masi AT, McShane DJ, Rothfield NF, et al. The 1982 revised criteria for the classification of systemic lupus erythematosus. Arthritis Rheum. 1982; 25:1271-7. [PubMed: 7138600]

12. Dall'era M, Stone D, Levesque V, Cisternas M, Wofsy D. Identification of biomarkers that predict response to treatment of lupus nephritis with mycophenolate mofetil or pulse cyclophosphamide. Arthritis Care Res. 2011; 63:351-7.

13. Esmon CT, Esmon NL. The link between vascular features and thrombosis [review]. Annu Rev Physiol. 2011; 73:503-14. [PubMed: 20887194]

14. Redl H, Schlag G, Schiesser A, Davies J. Thrombomodulin release in baboon sepsis: its dependence on the dose of Escherichia coli and the presence of tumor necrosis factor. J Infect Dis. 1995; 171:1522-7. [PubMed: 7769287]

15. Delvaeye M, Noris M, De Vriese A, Esmon CT, Esmon NL, Ferrell G, et al. Thrombomodulin mutations in atypical hemolyticuremic syndrome. N Engl J Med. 2009; 361:345-57. [PubMed: 19625716]

16. Lichtnekert J, Rupanagudi KV, Kulkarni OP, Darisipudi MN, Allam R, Anders HJ. Activated protein $\mathrm{C}$ attenuates systemic lupus erythematosus and lupus nephritis in MRL-Fas(lpr) mice. J Immunol. 2011; 187:3413-21. [PubMed: 21849682]

17. Schiffer L, Bethunaickan R, Ramanujam M, Huang W, Schiffer M, Tao H, et al. Activated renal macrophages are markers of disease onset and disease remission in lupus nephritis. J Immunol. 2008; 180:1938-47. [PubMed: 18209092]

18. Stearns-Kurosawa DJ, Kurosawa S, Mollica JS, Ferrell GL, Esmon CT. The endothelial cell protein $\mathrm{C}$ receptor augments protein $\mathrm{C}$ activation by the thrombin-thrombomodulin complex. Proc Natl Acad Sci USA. 1996; 93:10212-6. [PubMed: 8816778]

19. Riewald M, Ruf W. Protease-activated receptor-1 signaling by activated protein C in cytokineperturbed endothelial cells is distinct from thrombin signaling. J Biol Chem. 2005; 280:19808-14. [PubMed: 15769747]

20. Volpert OV, Zaichuk T, Zhou W, Reiher F, Ferguson TA, Stuart PM, et al. Inducer-stimulated Fas targets activated endothelium for destruction by anti-angiogenic thrombospondin-1 and pigment epithelium-derived factor. Nat Med. 2002; 8:349-57. [PubMed: 11927940] 


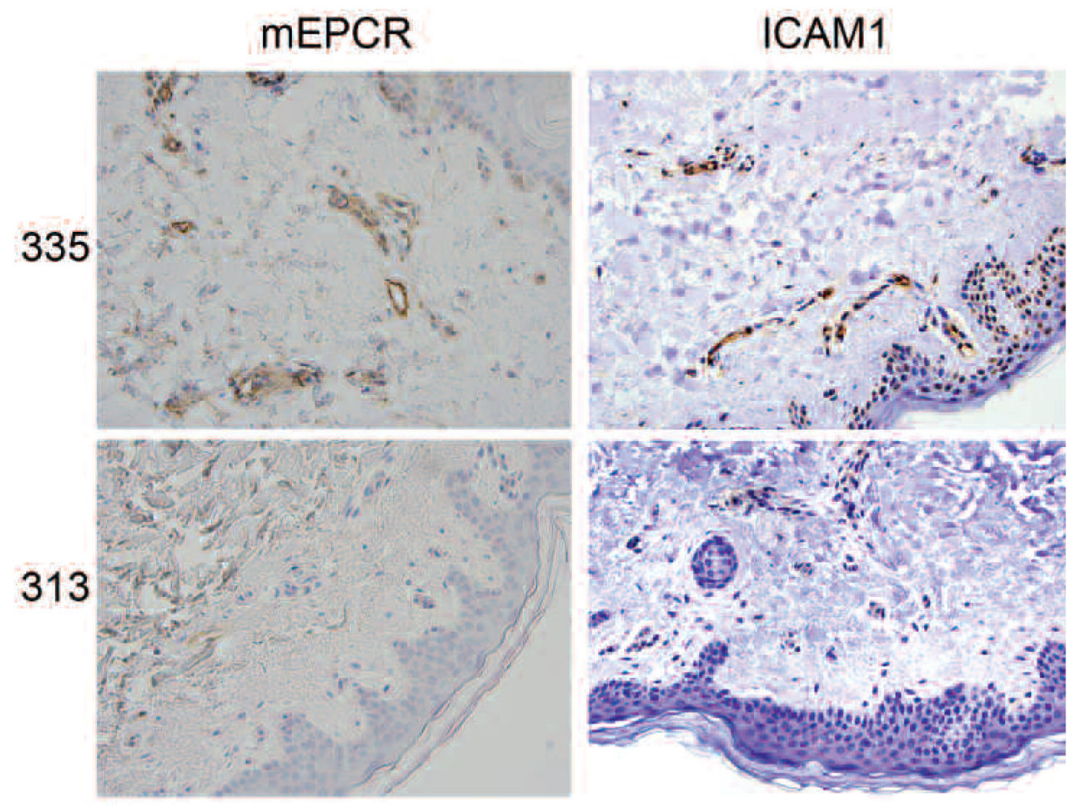

Figure 1.

Immunohistochemical staining for membrane endothelial protein $\mathrm{C}$ receptor $(\mathrm{mEPCR})$ and intercellular adhesion molecule-1 (ICAM-1) in nonlesional non-sun-exposed skin sections from a patient with Class IV lupus nephritis (Patient 335) and a healthy control (Subject 313). Sections of skin from patient and control were immunostained with anti-EPCR monoclonal antibody or anti-ICAM-1 and counterstained with hematoxylin. Positive "brown" blood vessels and negative blood vessels are evident in both cases. Regarding the immunohistochemistry result for mEPCR, diffuse immunostaining of mEPCR-positive blood vessels is visible for antigen in the patient sample while there is a focal staining pattern in the control skin section. A similar trend was observed for the immunohistochemistry result for ICAM-1. 


\section{Table 1}

Clinical data and dermal endothelial staining results in patients with SLE compared to controls.

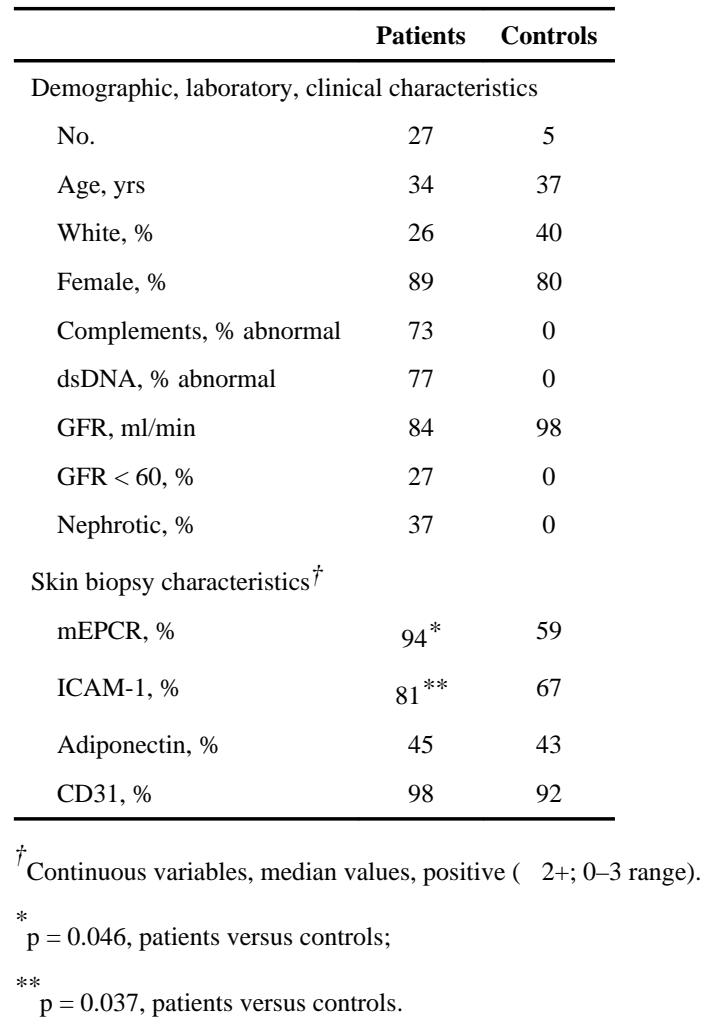

GFR: glomerular filtration rate; mEPCR: membrane endothelial protein C receptor; ICAM-1: intercellular adhesion molecule-1; SLE: systemic lupus erythematosus. 
